\title{
In vitro maturation of bovine oocytes: beneficial effects of cysteamine
}

\begin{abstract}
Antioxidant substances used at any stage of in vitro embryo production increase intracellular glutathione synthesis (GSH), improve nuclear maturation rates and protect embryos against endogenous or exogenous oxidative stresses, making embryos resistant to freeze, as well as increasing cell quality and number of embryos reaching blastocysts. Production of GSH depends on the availability and uptake of cysteine in the medium. However, cysteine is very unstable outside the cell and is auto-oxidized to cystine. Cysteamine reduces cystine to cysteine and promotes the uptake of cysteine by cells thereby enhancing the GSH synthesis. Consequently, cysteamine plays an important role in the synthesis of GSH and is a key factor in the defense mechanism against ROS.
\end{abstract}

Keywords: bovine, oocyte, maturation, culture, cysteamine
Volume 7 Issue 2 - 2018

\section{Asiye Izem Sandal, Ozen Banu Ozdas, Alper Baran}

Department of Reproduction and Artificial Insemination, Istanbul University, Turkey

Correspondence: Asiye Izem Sandal, DVM PhD, Istanbul University, Faculty of Veterinary Medicine, Department of Reproduction and Artificial Insemination, 34320, Avcilar, Istanbul, Turkey,Tel +90 212473 7070-1723, Email izem@istanbul.edu.tr

Received: April II, 2017 | Published: May 02, 2018

\section{Introduction}

Antioxidant and cryoprotectant substances has often been supplemented to media for in vitro production and vitrification of bovine embryos in the last ten years. ${ }^{1-3}$ It has been established that oxygen pressure in oviduct and uterus is less than atmospheric oxygen pressure. ${ }^{4}$ In vitro embryo production of mammals under atmospheric oxygen pressure is being used routinely but during embryo culture, this high pressure leads to the formation of reactive oxygen species (ROS). ${ }^{5-7}$ Harmful effects of ROS are DNA damage, lipid peroxidation, oxidative modifications of proteins, spermatozoon and oocyte fusion inhibition. ${ }^{8}$ As well as known negative effects of ROS in some circumstances cell apoptosis is another important physiological factor. ${ }^{5,9}$ One of the most important endogen sources of ROS is oxidative phosphorylation. Inhibition of oxidative phosphorylation decreases ROS and has a positive effect on in vitro embryo development. ${ }^{10}$ The most important factor that leads to an increase in the formation of ROS is the exogenous oxygen pressure. Oxygen pressure in the oviduct is only $1 / 4^{\text {th }}$ of atmospheric oxygen pressure. In in vitro produced bovine embryos under low oxygen tension $(5-7 \%)$ it has been reported to increase resistance to freezing and non-protein structure one of the sulfhydryl compounds glutathione (GSH) shynthesis. ${ }^{11,12}$ Oxidative stress is mediated by reactive oxygen species and results in an imbalance of the intracellular redox potential. ${ }^{13}$ Reactive oxygen species are highly reactive and unstable. They can react with nucleic acids, lipids, proteins, carbohydrates to acquire an electron and become stable. These reactions induce a cascade of subsequent chain reactions eventually resulting in cellular damage. ${ }^{14-16}$ Antioxidants such as $\beta$-mercaptoethanol, cysteamine, cystine, cysteine, N-acetyl-L-cysteine (NAC), superoxide dismutase (SOD) and resveratrol are used frequently in order to protect in vitro produced bovine embryos against oxidative stress. ${ }^{6,17,18}$ It is known that antioxidants have positive effects on embryo development but some researchers advocate these positive effects can be effective under certain conditions. ${ }^{9}, 19$ Studies revealed that positive effects of antioxidants can be occurred fewer than $20 \%$ oxygen tension. ${ }^{19}$
Various oxygen pressures have been tested in different culture conditions by researcher's in vitro production of bovine embryos. For example, oviductal or granulosa cells used in the co-culture environments $20 \% \mathrm{O}$ pressure, non-co-culture that does not contain somatic cells environments $5 \%$ oxygen pressure was found to increase results. ${ }^{9,20}$ So many researchers advocate antioxidant substances have positive effects on oocyte maturation also embryo development but the mechanism of antioxidant action is not well known. ${ }^{21,22}$ In the studies without supplementing antioxidant substances maturated and cultured embryos in different animal species and medium reaching to blastocyst stage in bovine in TCM-199 medium is 7.2\%, in SOF medium $6 \%$ also in bovine, in pigs it was detected $23.3 \%$ as in SOF medium. ${ }^{11,17,23,24}$ Gasparrini et al., ${ }^{24}$ detected cleavage rate of bovine embryos as $56.92 \%$ that was supplemented with $100 \mu \mathrm{M}$ cysteamine in TCM-199 medium for maturation. Singhal et al. ${ }^{23}$ reported in their study the cleavage rate of the buffalo oocytes as $60.7 \%$ supplemented with $50 \mu \mathrm{M}$ cysteamine. Oyamada \& Fukui ${ }^{11}$ investigated the effect of epidermal growth factor and cysteamine on the maturation of bovine oocytes, in the group that they added $100 \mu \mathrm{M}$ cysteamine, they found the cleavage rate as $62.4 \%$, in the group they added epidermal growth factor and cysteamine, and they found the cleavage rate as $63.2 \%$. Low molecular weight thiol components such as cysteine and cystine are precursors of glutathione (GSH), which plays an important protective role in relation to ROS generated by normal oxidative metabolism in the cell. The cellular content of GSH is regulated by the gammaglutamyl cycle as reviewed. ${ }^{25}$ Production of GSH depends on the availability and uptake of cysteine in the medium. However, cysteine is very unstable outside the cell and is auto-oxidized to cystine. Cysteamine reduces cystine to cysteine and promotes the uptake of cysteine by cells thereby enhancing the GSH synthesis. Consequently, cysteamine plays an important role in the synthesis of GSH and is a key factor in the defense mechanism against ROS.

Antioxidant substances used at any stage of in vitro embryo production increase intracellular glutathione synthesis (GSH), improve nuclear maturation rates and protect embryos against endogenous or 
exogenous oxidative stresses, making embryos resistant to freeze, as well as increasing cell quality and number of embryos reaching blastocysts. Increased cell quality, more resistant to freeze, and an increase in the number of embryos that can be transferred are undoubtedly desirable. Scientists are doing a myriad of research to ensure that in vitro produced embryos can both be transferred and frozen. They work to standardize or improve existing outcomes using different chemistries, methods, either in the in vitro production phase, transfer, pregnancy and birth, as well as freezing stages. In some studies, it has been reported that antioxidants and transfer methods used in the in vitro production stage of the embryo may contribute to the development of genetically superior embryos and also to the spread of the embryo transfer technique. In contrast to these results, the embryos from oocytes exposed to cysteamine did not appear to be different with respect to cryotolerance, post-transfer embryo survival, and calf, ovine characteristics as measured by gestation length, birth weight, perinatal mortality, and sex ratio..$^{26,27}$

\section{Conclusion}

Low molecular weight thiol compounds, such as cysteamine, can increase cysteine uptake by oocytes during IVM. This subsequently can increase GSH content, which is a major anti-oxidant system that protects the cells against the deleterious effects of oxidative stress by scavenging ROS.

\section{Acknowledgments}

None.

\section{Conflict of interest}

Authors declare that there is no conflict of interest.

\section{References}

1. Nedambale TL, Du F, Yang X, et al. Higher survival rate of vitrified and thawed in vitro produced bovine blastocysts following culture in defined medium supplemented with $\boldsymbol{\beta}$-mercaptoethanol. Anim Reprod Sci. 2006;93(1-2):61-75

2. Manjunatha BM, Gupta PSP, Ravindra JP, et al. Effect of vitrification medium composition and exposure time on post-thaw development of buffalo embryos produced in vitro. Vet J. 2009;179:287-291.

3. Saragusty J,AravA. Current progress in oocyte and embryo cryopreservation by slow freezing and vitrification. Reproduction. 201;141(1):1-19.

4. Fischer B, Bavister BD. Oxygen tension in the oviduct and uterus of rhesus monkeys, hamsters and rabbits. J Reprod Fertil. 1993;99(2):673-679.

5. Van Soom A, Yuan YQ, Peelman LJ, et al. Prevalence of apoptosis and inner cell allocation in bovine embryos cultured under different oxygen tensions with or without cysteine addition. Theriogenology. 2001;57(5):1453-1465.

6. Ali AA, Bilodeau JF, Sirard MA. Antioxidant requirements for bovine oocytes varies during in vitro maturation, fertilization and development. Theriogenology 2003;59(3-4):939-949.

7. Kitagawa Y, Suzuki K, Yoneda A, et al. Effects of oxygen concentration and antioxidants on the in vitro developmental ability, production of reactive oxygen species (ROS), and DNA fragmentation in porcine embryos. Theriogenology. 2004;62(7):1186-1197.

8. Aitken RJ, Harkiss D, Buckingham D. Relationship between ironcatalysed lipid peroxidation potential and human sperm function. $J$ Reprod and Fertil. 1993;98(1):257-265.
9. Yuan YQ, Van Soom A, Coopman FOJ, et al. Influence of oxygen tension on apoptosis and hatching in bovine embryos cultured in vitro. Theriogenology. 59(7):1585-1596.

10. Thompson JG, McNaughton C, Gasparrini B, et al. Effects of inhibitors and uncouplers of oxidative phosphorylation during compaction and blastulation of bovine embryos cultured in vitro. $J$ Reprod Fertil. 2000;118(1):47-55.

11. Oyamada T, Fukui Y (2004) Oxygen tension and medium supplements for in vitro maturation of bovine oocytes cultured individually in a chemically defined medium. J Reprod Dev. 2004;50(1):107-117.

12. Raty M, Ketoja E, Pitkanen T, et al. In vitro maturation supplements affect developmental competence of bovine cumulus-oocyte complexes and embryo quality after vitrification. Cryobiology. 2011;63(3):245-255.

13. Balaban RS, Nemoto S, Finkel T. Mitochondria, oxidants, and aging. Cell. 2005;120(4):483-495.

14. Attaran M, Pasqualotto E, Falcone T, et al. The effect of follicular fluid reactive oxygen species on the outcome of in vitro fertilization. Int J Fertil Womens Med. 2000;45(5):314-320.

15. Szczepanska M, Kozlik J, Skrzypczak J, et al. Oxidative stress may be a piece in the endometriosis puzzle. Fertil Steril. 2003;79(6):1288-1293.

16. Pierce JD, Cackler AB, Arnett MG. Why should you care about free radicals? $R N$. 2004;67(1):38-42.

17. Balasubramanian S, Rho GJ. Effect of cysteamine supplementation of in vitro matured bovine oocytes on chilling sensitivity and development of embryos. Anim Reprod Sci. 2007;98(3-4):282-292.

18. Kobayashi M, Lee ES, Fukui Y. Cysteamine or $\boldsymbol{\beta}$-mercaptoethanol added to defined maturation medium improves blastocyst formation of porcine oocytes after intracytoplasmic sperm injection. Theriogenology. 2006;65(6):1191-1199.

19. Olson SE, Seidel GE. Culture of in vitro-produced bovine embryos with vitamin $\mathrm{E}$ improves development in vitro and after transfer to recipients. Bio Reprod. 2000;62(2):248-252.

20. Ozdas OB, Baran A, Tas M, et al. Effect of different transport temperatures on in vitro maturation of oocytes collected from frozen-thawed sheep ovaries. Turk J Vet Anim Sci. 2013;36(1):1104-1124.

21. Sandal AI, Ozdas OB. Vitrification of in-vitro produced bovine embryos matured in modified TCM-199. Turk J Vet Anim Sci. 2015;39:688-692.

22. Enginler SO, Ozdas OB, Sandal AI, et al. The effect of cysteamine and oviductal cells in different culture media on the development of sheep embryos. J F Vet Med Kafkas Uni. 2016;22(1):139-145.

23. Singhal S, Prasad S, Singh B, et al. Effect of including growth factors and antioxidants in maturation medium used for in vitro culture of buffalo oocytes recovered in vivo. Anim Reprod Sci. 2009;113(1-4):44-50.

24. Gasparrini BF, Neglia G, Di Palo R, et al. Effect of Cysteamine during in vitro maturation on buffalo embryo development. Theriogenology. 2000;54(9):1537-1542.

25. Deleuze S, Goudet G. Cysteamine supplemantation of in vitro maturation media: a review. Reprod Dom Anim. 2010;45(6):476-482.

26. Dos Santos-Neto PC, Cuadro F, Barrera N, et al. Embryo survival and birth rate after minimum volume vitrification or slow freezing of in vivo and in vitro produced ovine embryos. Cryobiology. 2017;78:8-14.

27. Merton JS, Knijn HM, Flapper H, et al. Cysteamine supplementation during in vitro maturation of slaughterhouse- and opu-derived bovine oocytes improves embryonic development without affecting cryotolerance, pregnancy rate, and calf characteristics. Theriogenology. 2013;80(4):365371. 